\title{
Epigenetic regulation and chromatin remodeling in learning and memory
}

\author{
Somi Kim and Bong-Kiun Kaang
}

Understanding the underlying mechanisms of memory formation and maintenance has been a major goal in the field of neuroscience. Memory formation and maintenance are tightly controlled complex processes. Among the various processes occurring at different levels, gene expression regulation is especially crucial for proper memory processing, as some genes need to be activated while some genes must be suppressed. Epigenetic regulation of the genome involves processes such as DNA methylation and histone post-translational modifications. These processes edit genomic properties or the interactions between the genome and histone cores. They then induce structural changes in the chromatin and lead to transcriptional changes of different genes. Recent studies have focused on the concept of chromatin remodeling, which consists of 3D structural changes in chromatin in relation to gene regulation, and is an important process in learning and memory. In this review, we will introduce three major epigenetic processes involved in memory regulation: DNA methylation, histone methylation and histone acetylation. We will also discuss general mechanisms of long-term memory storage and relate the epigenetic control of learning and memory to chromatin remodeling. Finally, we will discuss how epigenetic mechanisms can contribute to the pathologies of neurological disorders and cause memory-related symptoms.

Experimental \& Molecular Medicine (2017) 49, e281; doi:10.1038/emm.2016.140; published online 13 January 2017

\section{INTRODUCTION}

Many studies have shown evidence of active epigenetic marker changes during learning and memory processes. ${ }^{1,2}$ The term 'neuroepigenetics' describes memory processes as consequences of dynamic experience-dependent changes in the genome. ${ }^{3}$ Epigenetic mechanisms cause DNA compaction and relaxation, which lead to transcriptional repression and activation, respectively. Chromatin is made of histone units, with each unit composed of an 8-subunit histone core and the DNA coiling around it. ${ }^{4}$ As 146 bps of DNA coil around one histone, DNA is compacted and is able to fit into the nucleus. Chromatin may adopt one of two major states in an interchangeable manner. These states are heterochromatin and euchromatin. Heterochromatin is a compact form that is resistant to the binding of various proteins, such as transcriptional machinery. In contrast, euchromatin is a relaxed form of chromatin that is open to modifications and transcriptional processes (Figure 1$)^{5}$.

The term epigenetics was coined by Waddington in 1942, and was used to describe the 'interactions of genes with their environment that brings the phenotype into being' ${ }^{6}$
Waddington originally used the term epigenetics to explain the phenomena in which changes not encoded in the DNA occur in the cell during development in response to environmental stimuli. Since then, an extensive number of studies has shown that long-lasting epigenetic changes occur in the genomes of cells. These changes include changes to post-mitotic neurons, which are used to incorporate experience-dependent changes. ${ }^{7}$ An early study showing the important relationship between epigenetics and synaptic plasticity is that of Kandel and colleagues. This study investigated long-term effect of excitatory and inhibitory signaling in Aplysia sensory neurons. The authors discovered that the facilitatory transmitter 5-HT activates cyclic AMPresponsive element-binding protein 1 , which causes histone acetylation. On the other hand, the inhibitory transmitter FMRFa causes CREB2 activation and histone deacetylation. ${ }^{8}$ These results indicate that gene expression and epigenetic changes are required for long-term memory-related synaptic plasticity in Aplysia. Thus, epigenetic modifications are made independently of actual genome sequence changes and lead to the creation of the 'epigenome.' The epigenome undergoes

Department of Biological Sciences \& Brain and Cognitive Sciences, College of Natural Sciences, Seoul National University, Seoul, Republic of Korea Correspondence: Professor B-K Kaang, Department of Biological Sciences \& Brain and Cognitive Sciences, College of Natural Sciences, Seoul National University, Building 504 Rm 202, 1 Gwanangno, Gwanak-gu, Seoul 08826, Republic of Korea.

E-mail: kaang@snu.ac.kr

Received 16 July 2016; revised 21 September 2016; accepted 25 September 2016 

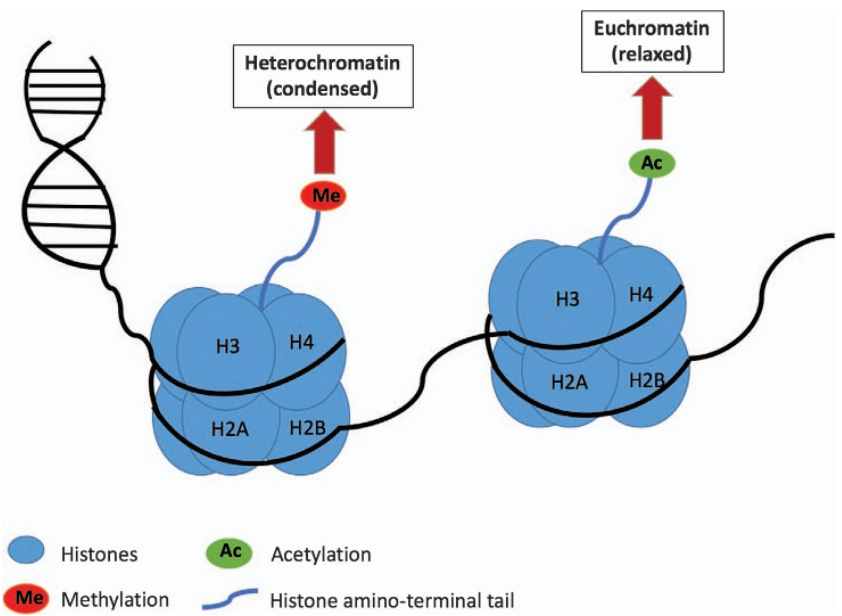

Figure 1 Schematic drawing of histone methylation and acetylation in relation to chromatin remodeling. Addition of methyl groups to the tails of histone core proteins leads to histone methylation, which in turn leads to the adoption of a condensed state of chromatin called 'heterochromatin.' Heterochromatin blocks transcription machinery from binding to DNA and results in transcriptional repression. The addition of acetyl groups to lysine residues in the $\mathrm{N}$-terminal tails of histones causes histone acetylation, which leads to the adoption of a relaxed state of chromatin called 'euchromatin.' In this state, transcription factors and other proteins can bind to their DNA binding sites and proceed with active transcription.

biochemical changes in response to environmental stimuli and leads to the remodeling of chromatin structure. The term chromatin remodeling mainly refers to the ATP-dependent process of genomic transformation by enzymes that shift nucleosomes, such as the SWI/SNF complex. ${ }^{9}$ This mechanistic change in genomic conformation sets out a platform for various cellular processes, such as the exposure of the promoter of a specific gene to its transcriptional machinery. ${ }^{9}$ Chromatin remodeling, DNA methylation and histone posttranslational modifications (PTMs) are all known to be important long-term memory processes. There are many types of histone modifications, such as acetylation, methylation, phosphorylation, ubiquitination and ADP-ribosylation. ${ }^{10}$ In this review, we will discuss DNA methylation, histone modifications and chromatin remodeling, which are the most studied epigenetic mechanisms in relation to memory processes.

\section{MEMORY FORMATION AND STORAGE}

Memory allows us to acquire new information and to store it in our brains. Memory enables animals to behave adaptively to changing environments. While memory formation and storage require many processes at the cellular level, the "memory engram' can be considered to be a biological trace of memory. This term was first coined by Richard Semon and denoted the 'hypothetical material basis of learned information.. ${ }^{11}$ Memory engram-bearing cells are specific cells that retain biochemical changes induced by learning and sustain the information until it is later retrieved based on appropriate cues. ${ }^{11}$ How experiences are transformed into the memory engram and stored for a long time is now an extensively studied field in neuroscience.

After a memory is formed, a chain of biological reactions occurs to store it for the long term. Roberson and Sweatt described these reactions as 'mnemogenic,' which is a term based on the Greek word for memory formation. ${ }^{12}$ Mnemogenic reactions, such as de novo protein synthesis and DNA-histone modifications, chemically alter the biological system so that the acquired information is stably protected from protein turnover. ${ }^{13}$ Another important aspect of memory is the change in synaptic connection strength. This phenomenon is called long-term potentiation (LTP), during which synaptic connections are strengthened and synaptic efficacy is increased. ${ }^{14}$ Bliss and Lomo described LTP for the first time in 1973 through an experiment that showed that a train of high-frequency stimulation causes an increase in synaptic transmission efficiency in the rabbit brain. This synaptic strengthening was effective for several hours and required a number of biological changes. ${ }^{15}$ On the postsynaptic side, glutamate signaling through $\alpha$-amino-3-hydroxy-5-methyl-4isoxazolepropionic acid and $N$-methyl-D-aspartate receptors trigger $\mathrm{Ca}^{2+} /$ calmodulin-dependent protein (CaMKII) activation. CaMKII is a well-known protein that triggers secondary signaling processes that play central roles in promoting synaptic plasticity. ${ }^{16}$ The rhythmic bursts of activity that induce LTP mimic the naturally occurring theta rhythm that is present in the hippocampus during learning. ${ }^{17}$ Several studies have shown that injecting LTP blockers, such as CaMKII inhibitors and extracellular signal-regulated kinase inhibitor into the hippocampus cause impairments in hippocampus-dependent tasks, such as the Morris water maze. ${ }^{16,18}$ There are two major types of memories: short-term memories, which last for a few hours, and long-term memories, which persist for several days or longer. Long-term memory formation requires a cascade of processes, which are needed to produce new messenger RNAs and proteins related to synaptic plasticity. ${ }^{19}$ When dendritic spines were imaged over different time courses in different regions of the mouse brain, the fraction of spines undergoing turnover persistently decreased. This shows that synaptic connections are continually stabilized during adulthood. In addition, spine turnover rates were different in the different brain regions. This indicates that different brain regions differ in their capacities for experience-dependent plasticity. ${ }^{20}$ The fact that the plasticity of a neuron can be altered for a long time can be explained by the synthesis of new proteins in the soma. Active transcription and local translation aid the maintenance of LTP and memory consolidation (Figure 2).

To be stably stored, a memory must overcome its vulnerability to external disruptions. After initial acquisition, memory transforms from a transient state into a stable state during 'memory consolidation'. ${ }^{21}$ Cellular and circuit level structural changes support long-term memory storage and involve different brain regions and networks. After fear conditioning training, the mouse hippocampus and anterior cingulate cortex exhibit dendritic spine growth in a time-dependent manner. ${ }^{22}$ How memories persist for a lifetime may be explained by 


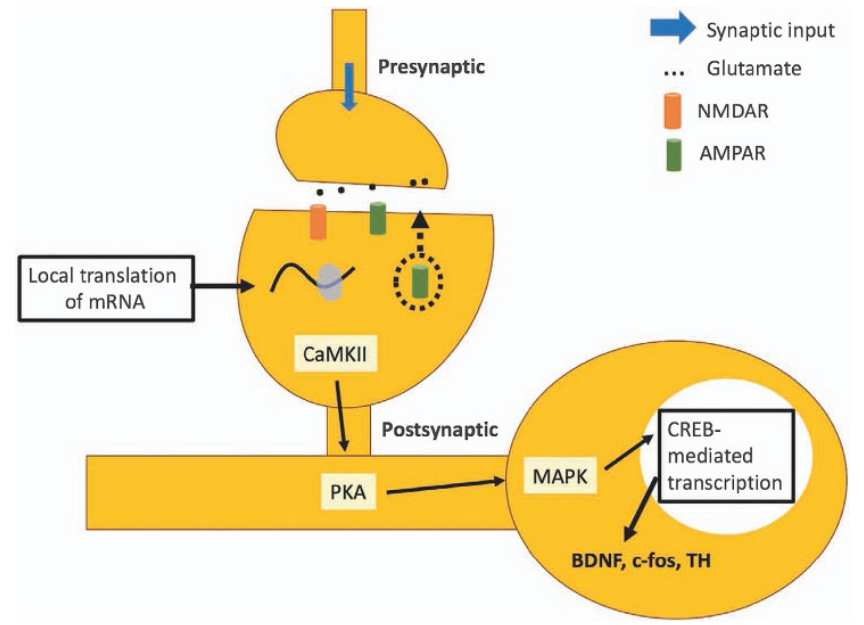

Figure 2 Illustration of late-LTP (L-LTP)_mechanism. Memory allocation involves several brain regions, including the hippocampus. As neurons in this region are recruited to store the memory trace, hippocampal CA1 region pyramidal cells undergo structural changes at the dendritic level and form new synaptic connections. Synaptic transmission involves pre-synaptic glutamate release in the gap junction and post-synaptic $\mathrm{N}$-methyl-D-aspartate and AMPA receptor activation and downstream signaling cascades. The influx of $\mathrm{Ca}^{2+}$ through $\mathrm{N}$-methyl-D-aspartate receptors into the cytoplasm activates CaMKII, which then activates protein kinase $A$ (PKA) and mitogen-activated protein kinase (MAPK). Activated MAPK then induces CREB-mediated transcription in the nucleus, as CREB is a transcription factor that can lead to the synthesis of proteins, such as BDNF, c-fos and tyrosine hydroxylase. At dendritic sites, local translation independent of somatic transcription is also important for the enhancement of synaptic strength. Altogether, newly synthesized proteins are involved in processes such as receptor trafficking and cytoskeletal scaffolding, which contribute to AMPA receptor insertion and neurotransmitter responsiveness. These morphological changes enable L-LTP maintenance, which is thought to be the cellular basis for memory storage.

epigenetic regulation mechanisms. Chromatin transitions between the two states are mainly governed by DNA methylation and PTMs of histones. A study by Sweatt and his colleagues indicates that DNA methyltransferase (Dnmt) gene expression is increased in the adult rat hippocampus after contextual fear conditioning. ${ }^{23}$ The authors also found that methylation of protein phosphatase 1 (PP1), a memory repressor gene, is increased after fear conditioning, while the synaptic plasticity gene reelin is demethylated and transcribed. Cortical DNA methylation is known to be important for remote memory formation. For example, the increased methylation of the memory-suppressor gene, Calcineurin $(\mathrm{CaN})$, in cortical neurons persisted up to 30 days after fear conditioning. Furthermore, its messenger RNA levels were reduced in the training group compared to the control group. Infusion of DNMT inhibitors into the anterior cingulate cortex disrupted fear memory and reduced $\mathrm{CaN}$ methylation levels. ${ }^{24}$ These results support the idea that epigenetic changes, such as DNA methylation induced by experience, represent long-lasting traces of memory.

\section{DNA METHYLATION AND MEMORY REGULATION}

DNA methylation is known to occur on nucleotide base cytosines that are next to guanine $(\mathrm{CpG})$ and regulates chromatin state transitions. During DNA methylation, DNMTs aid the covalent binding of a methyl group from S-adenosylmethionine to the $5^{\prime}$ position of cytosine. ${ }^{25} \mathrm{DNA}$ methylation typically represses transcription by inhibiting the binding of the transcriptional machinery to binding sites. DNA methylation is often referred to as a lifelong inhibitory change that induces gene silencing. DNA methylation in neuronal cells can persist because mature neurons do not divide. Furthermore, if a methylated cytosine is damaged, the new cytosine is methylated to reconstruct the epigenetic change. ${ }^{13}$ Thus, we can say that DNA methylation, like other epigenetic processes, self-perpetuates through DNMT activity.

There is extensive evidence that DNA methylation is regulated in an experience-dependent manner by neural activity. An early discovery indicating the involvement of active DNA methylation in hippocampus-dependent memory showed that neuronal activation induces sufficient changes in the DNA methylation of memory-related genes. Treating with DNMT blockers inhibits memory formation and synaptic plasticity induction. ${ }^{26}$ There are three types of DNMTs: DNMT1, DNMT3a and DNMT3b. Before the discovery of the demethylation activity of Tet enzymes, it was once thought that DNA methylation was irreversible and that demethylation is unlikely to occur in mature neurons. ${ }^{27}$ However, this idea was proven wrong by work showing that Tet enzymes have active demethylation activity and affect memory formation and stabilization. ${ }^{28}$ Tet1 knockout (KO) transgenic mice have impaired gene expression and memory extinction. ${ }^{29}$ In addition, when DNA methylation was blocked in the anterior cingulate cortex 30 days after memory formation by inhibiting DNMTs using drugs, remote memory was impaired. ${ }^{24}$ These data imply the presence of important roles for DNA methylation and demethylation in memory maintenance and extinction.

How does DNA methylation regulate gene transcription? A pair of methyl groups at the major groove of DNA blocks transcription factors from recognizing their binding sites. DNA methylation also increases chromatin compaction by attracting histone-remodeling enzymes. Histone-remodeling proteins are attracted to methylated DNA sites by methyl CpG-binding domain (MBD) proteins. MBD proteins recruit transcriptional corepressors, such as histone deacetylases, which increase the positive charge of the nucleosome and alter local chromatin structure into the transcriptionally incompetent heterochromatin state $^{30,31}$ Mutations in MBD can cause developmental disabilities, such as Rett syndrome, which is caused by an MBD mutation in the MECP2 protein. ${ }^{32}$

Cognitive ability tends to decline with age. Oliveira et al. ${ }^{33}$ found that cognitive decline associated with aging is concomitant with a reduction in DNA methyltransferase Dnmt3a2 expression in the hippocampus. When the authors restored Dnmt3a2 levels in aged mice, cognitive ability was restored to normal levels in tasks such as trace fear conditioning. 
In another study, fear learning caused DNA methylation changes in different brain regions, as DNMT3a and $3 b$ expression in the hippocampus was increased after contextual fear conditioning. Furthermore, while histone H3 acetylation and DNMT3a expression were increased in the lateral amygdala after auditory fear conditioning. ${ }^{34,35}$ Blocking DNMT pharmacologically or using the intra-CA1 infusion of inhibitors such as 5-azadeoxycytidine or zebularine resulted in impairment of contextual fear conditioning. ${ }^{23,36}$ These data appear to be rather controversial because DNA methylation has been known to negatively regulate memory. However, recent evidence suggests that DNA methylation is involved in silencing the expression of memory suppressor genes, such as PP1, and that DNA methylation may regulate memory by interacting with histone acetylation and changing its levels. ${ }^{34}$ Although further investigations are needed to clarify the roles of DNA methylation in memory consolidation, a body of evidence clearly indicates that memory formation requires hypermethylation of memory suppressor genes and hypomethylation of memory promoting genes. Thus, DNA methylation is dynamically regulated in memory processes. ${ }^{13}$

\section{PTMS OF HISTONES}

In addition to DNA methylation, post-translational histone modifications are also important for chromatin geometry and gene expression. ${ }^{37}$ The positive charges of unmodified histone proteins promote a tight interaction with negatively charged DNA and cause chromatin to be in a transcriptionally unfavorable state. However, histones undergo modifications, including acetylation, phosphorylation and methylation, that alter their charges and binding properties. ${ }^{38}$ Histone acetylation involves the histone acetyltransferase (HAT) enzyme. It is often associated with transcriptional activation, as acetylated histones act as binding sites for transcriptional machinery. Histone phosphorylation is also associated with transcriptional activation, while histone methylation can promote both transcriptional activation and repression. ${ }^{39}$ Newly formed hippocampus-dependent memories need to be stabilized into a persistent memory trace. Mice with decreased PP1 levels had enhanced remote memory performance with increasing histone PTMs. ${ }^{40}$ This suggests that histone PTMs are important for memory consolidation and retention. The levels of PTMs of histones associated with the promoter region of Zif268, which is an immediate early gene involved in memory, were shown to increase and their expression was shifted from the hippocampus to the prefrontal cortex as memories matured. ${ }^{41}$ The above data show the important roles of histone PTMs in different brain regions and how they facilitate memory consolidation.

Increasing histone acetylation levels by blocking the HDAC can cause an increase in memory storage. Therefore, altering histone modifications or the activity of histone-modifying enzymes, including CREB binding-protein (CBP), affect memory storage. ${ }^{42}$ Histone modifications may gate the transcription of plasticity genes that change the response of individual neurons and regulate behavior. These histone modification patterns may alter the structure of chromatin and its interaction interface for transcriptional proteins.

\section{HISTONE ACETYLATION IN MEMORY FORMATION}

Among the various types of histone modifications, histone acetylation is one of the most studied mechanisms. It involves the addition of an acetyl group to a lysine present at the $\mathrm{N}$-terminus tail of the nucleosome, which is the basic unit of DNA packaging in eukaryotic cells. It was once assumed that histone acetylation leads to a charge neutralization in the nucleosome, which in turn causes transcriptional facilitation. ${ }^{43}$ However, a number of later studies indicated that the recognition of an acetylated lysine by transcriptional proteins is more important than the change in the charge itself. ${ }^{44}$ Histone acetylation is rapid and reversible, as is controlled by the activities of HAT and HDAC. HATs are often transcriptional coactivators that contain bromodomains, whereas histone deacetylase are corepressors. A bromodomain is comprised of about 110 amino acids that recognize acetylated lysines on the N-terminals of histone tails. ${ }^{45}$

It has been known for a long time that long-term memory formation requires de novo protein synthesis. ${ }^{46}$ It has also been shown that there exist critical time periods of protein synthesis after learning, ${ }^{47}$ indicating that there is a time limit for gene expression events for memory consolidation. Growing evidence indicates that histone acetylation is just as important for the persistence of long-term memory. HDAC inhibitors, such as like tyrosine $\mathrm{A}$ and sodium butyrate $(\mathrm{NaBu})$, enhance LTP in acute hippocampal slices and memory consolidation during contextual fear conditioning. ${ }^{39,48}$ Histone acetylation occurs at various lysine positions within the core histone. ${ }^{49}$ Therefore, it is possible that different forms of learning induce different patterns of acetylation at specific gene promoters. There are three main classes of HDAC based on sequence similarity to yeast proteins. ${ }^{50} \mathrm{HDAC} 2$ has been found to negatively regulate memory. When Guan et al. ${ }^{51}$ overexpressed HDAC2 in neurons, dendritic synaptic plasticity and memory formation were impaired, while Hdac2 deletion caused memory facilitation. CBP is a well-known transcriptional coactivator that has HAT activity and has been shown to be important for long-term memory formation. ${ }^{52,53}$ Mutations in CBP can contribute to the pathology of Rubinstein-Taybi syndrome, a neurodevelopment disorder characterized by cognitive impairment and characteristic facial features, such as microcephaly. ${ }^{54}$ The Bdnf promoter is known to be responsive to histone acetylation changes after learning. Bredy et al. ${ }^{55}$ found that fear conditioning leads to a distinct pattern of acetylation at histones $\mathrm{H} 3$ and $\mathrm{H} 4$ around $b d n f$ promoters. It has also been shown that CBP is recruited to the $c$-fos gene promoter in an activity-dependent manner, and that CBP's acetyltransferase activity is required for the expression of the $c$-fos gene, which is involved in memory formation and consolidation. ${ }^{56}$

\section{HISTONE METHYLATION}

Chromatin structure is also regulated through histone methylation. Although methylation is generally thought of as 
Table 1 List of neurological disorders caused by defects in epigenetic mechanisms

\begin{tabular}{llll}
\hline Disease & Symptom & Abnormal epigenetic mechanism & Reference \\
\hline Fragile X syndrome & Developmental and intellectual disabilities & DNA methylation \\
Rubinstein-Taybi syndrome & Broad thumbs and toes, and distinctive facial features & Histone acetylation \\
Alzheimer's disease & Neuronal loss and dementia & DNA methylation & 74 \\
Autism spectrum disorder & Deficits in social interaction and repetitive behavior & DNA methylation & 76 \\
Parkinson's disease & Resting tremor and postural instability & Histone acetylation & 77 \\
\hline
\end{tabular}

a transcriptional silencing marker, histone methylation can also induce transcriptional activation. Lysine can undergo mono-, di- and tri-methylation. Di- and tri-methylation of histone $\mathrm{H} 3$ at lysine $9(\mathrm{H} 3 \mathrm{~K} 9)$ is related to transcriptional repression, while methylation of histone $\mathrm{H} 3$ at lysine 4 (H3K4) is associated with transcriptional activation. ${ }^{57}$ In fact, Gupta et al. found that $1 \mathrm{~h}$ after contextual fear conditioning, $\mathrm{H} 3 \mathrm{~K} 4$ and $\mathrm{H} 3 \mathrm{~K} 9$ were both up-regulated. This suggests that active gene expression and repression through histone methylation are both necessary for memory formation. In addition, mice deficient in the H3K4-specific methyltransferase Mll exhibit a deficit in long-term contextual fear conditioning. This suggests that histone methylation is required for proper long-term consolidation of contextual fear memory. ${ }^{58}$

Histone methylation is controlled by enzymes called histone methyltransferases and histone demethylases. Histone methyltransferases belong to one of three enzyme families: PRMT1 arginine methyltransferases, SET-domain histone methyltransferases and non-SET-domain DOT1/DOT1L methyltransferases. ${ }^{59}$ Histone methylation can occur on arginine and lysine residues. However, lysine methylation on histones $\mathrm{H} 3$ and $\mathrm{H} 4$ is much more extensively studied. Treatment with the HDAC inhibitor $\mathrm{NaB}$ induces an enhancement in histone methylation in the hippocampus. This suggests a functional link between histone methylation and acetylation during fear memory consolidation. ${ }^{58}$ Kerimoglu et al. ${ }^{60}$ showed that mice deficient in the histone-methyltransferase myeloid/lymphoid or mixed-lineage leukemia 2 (mll2/kmt2b) gene in adult excitatory neurons show impairments in hippocampus-dependent memory tasks. DNA microarray data indicate that expression levels of 152 genes were downregulated in the dentate gyrus of $\mathrm{kmt} 2 \mathrm{~b}$-deficient mice. Bi-directional regulation of transcription based on cellular context and the quantity of methyl groups separates histone methylation from other epigenetic modifications, such as DNA methylation and histone acetylation, which mostly promote one direction of transcription. ${ }^{61}$ Histone methyltransferase inhibitors have recently been widely studied in preclinical trials aiming to develop drugs for cancer treatment. ${ }^{62}$ However, it is possible to use histone methylation as a therapeutic target for cognitive impairment by fixing the transcriptional discrepancy of memory-related genes. Impairments in histone lysine methylation are involved in many intellectual disability disorders, such as $9 \mathrm{q}$ subtelomere deletion syndrome. A mutation in the H3K9 methyltransferase EHMT1 is responsible for the pathology observed in $9 \mathrm{q}$ subtelomere deletion syndrome.
In fact, EHMT1 haploinsufficient mice exhibit intellectual disabilities and autistic phenotypes. ${ }^{61}$ Given the broad effects of histone methylation, generation of therapeutic drugs may be difficult and have a high risk of side effects. Therefore, selecting enzymes such as lysine methyltransferases (KMTs) and demethylases, which have relatively high target specificities, and precisely modifying a specific catalytic domain will be necessary. ${ }^{63}$

\section{CHROMATIN REMODELING}

It is clear that transcriptional regulation involves active interactions between transcription factors and chromatin. Chromatin structure is dynamic and precisely controls cellular processes, including gene expression. ${ }^{64}$ Taking a step back from investigating gene expression at the protein level, we find that global changes resulting from chromatin remodeling have received considerable attention in association with gene expression and memory processes. ${ }^{1}$ Chwang et al. ${ }^{65}$ revealed that the extracellular signal-regulated kinase-1/mitogen-activated protein kinase signaling pathway regulates histone phosphorylation and acetylation in the hippocampus after contextual fear conditioning and long-term memory formation. Their results support the idea that chromatin acts as a dynamic signal platform through active histone modifications. The aminoterminal tails of histone proteins subjected to post-translational modifications create a stage for dynamic interplay between histone and DNA modifications and the combinatorial possibilities for gene regulation. Chromatin structure is modified through two mechanisms. First, interactions between nucleosomes are broken. Second, various protein factors are recruited to the unraveled nucleosomes. ${ }^{66}$ There are various types of histone-modifying enzymes, such as acetyltransferases, methyltransferases, serine/threonine kinases, ubiquitin ligases and proline isomerases. Enzymes that carry out chromatin modifications and those that remove the modifications have been identified. For example, while G9a and CLL8 methylate lysine, enzymes such as LSD1/BHC110 and JDM1a have demethylase activity. Methyltransferases and kinases are the most characterized histone modifying enzymes. ${ }^{66}$ Learning and memory processes are associated with chromatin remodeling. Tsai and colleagues used CK-p25 transgenic (Tg) mice to show the critical effects of environmental enrichment (EE) on learning and memory. The CK-p25 Tg mouse is a neurodegenerative mouse model, in which the expression of the Alzheimer's disease-associated protein p25 is controlled by doxycycline. The authors showed that learning and long-term 
memory deficits in CK-p25 Tg mice are rescued following EE. This memory-restoring effect was achieved through EE-induced hippocampal and cortical chromatin remodeling, which leads to enhancements in synaptic efficacy. ${ }^{4}$

\section{CONCLUSION}

A few years ago, a group of scientists investigated the inheritance of parental experience using olfactory molecular specificity. They exposed F0 mice to acetophenone for odor fear conditioning and found that F1 and F2 generation mice similarly display an increased sensitivity to the F0-conditioned odor. Moreover, they found that sperm cells from F0 mice exhibit hypomethylation of the odorant receptor gene Olfr151 and that the F1 mice display neuroanatomical alterations of the olfactory system. These results indicate that experience-induced behavioral and structural adaptations can be biologically passed on to offspring through neuroanatomical and epigenetic changes. ${ }^{67}$ Mansuy and colleagues observed altered microRNA expression levels and trans-generational phenotype inheritance in mice that experience early life traumatic stress. They obtained similar results by injecting the traumatized males' sperm RNA into a fertilized wild type egg. Specifically, the resulting offspring exhibited similar behavioral and epigenetic characteristics to their traumatized parent. ${ }^{68}$

Epigenetic modifications can serve as enduring changes in the genome and help drive stable gene expression, which manifests as a long-lasting change in the animals' behavior. This may be accomplished by direct alteration of DNA or by post-translational modifications of histones. These modifications may include methylation, acetylation and phosphorylation. These covalent modifications affect the physical remodeling of chromatin structure or regulate recruitment of signaling complexes that drive or repress transcription. ${ }^{69}$ It is becoming increasingly clear that histone modifications and chromatin remodeling are critical for gene expression during memory processes. About $1-4 \%$ of mammalian genomic DNA consists of CpG dinucleotides and about $75 \%$ of these dinucleotides are known to be methylated. ${ }^{70}$

Failure of epigenetic processes can lead to pathogenesis characteristic of various neurological disorders (Table 1). One such example is Huntington's disease (HD), which is a fatal, inheritable disorder with a mid-life onset. HD patients show symptoms such as chorea, emotional distress and cognitive decline. HD is caused by a CAG repeat expansion in exon 1 of the huntingtin $(\mathrm{Htt})$ gene. Epigenetic modifications are found to be critical in HD pathogenesis. In patients with HD and HD transgenic mice, a number of genes, such as Sox2 and Pax6, were found to be hypermethylated, and to have accordingly reduced gene expression levels. ${ }^{71}$ Since these genes are important for neurogenesis, HD patients suffer from hippocampal neurogenesis deficits and cognitive decline. HDAC inhibitor treatment has been found to restore histone modification dysfunction and rescue some symptoms of HD. ${ }^{72}$ This study indicates that dysregulation of epigenetic processes can have prominent effects on the pathogeneses of neurological disorders. It is clear that epigenetic modifications and chromatin remodeling are necessary for proper memory formation and consolidation. As a number of chromatin-modifying drugs are being tested in preclinical studies, ${ }^{73}$ it will be important to extensively investigate the exact mechanisms of epigenetic regulation and to provide a basis for translational research and potential therapies for neurological diseases.

\section{CONFLICT OF INTEREST}

The authors declare no conflict of interest.

\section{ACKNOWLEDGEMENTS}

This work was supported by the National Honour Scientist Program grant (NRF-2012R1A3A1050385) to BKK and a BK21

fellowship to SK.

1 Levenson JM, Sweatt JD. Epigenetic mechanisms in memory formation. Nat Rev Neurosci 2005; 6: 108-118.

2 Peixoto L, Abel T. The role of histone acetylation in memory formation and cognitive impairments. Neuropsychopharmacology 2013; 38: 62-76.

3 Sweatt JD. The emerging field of neuroepigenetics. Neuron 2013; 80 : 624-632.

4 Fischer A, Sananbenesi F, Wang X, Dobbin M, Tsai LH. Recovery of learning and memory is associated with chromatin remodelling. Nature 2007; 447: 178-182.

5 Khalaf O, Graff J. Structural, synaptic, and epigenetic dynamics of enduring memories. Neural Plast 2016; 2016: 3425908.

6 Waddington CH. The epigenotype. 1942. Int J Epidemiol 2012; 41: 10-13.

7 Borrelli E, Nestler EJ, Allis CD, Sassone-Corsi P. Decoding the epigenetic language of neuronal plasticity. Neuron 2008; 60: 961-974.

8 Guan Z, Giustetto M, Lomvardas S, Kim J-H, Miniaci MC, Schwartz JH et al. Integration of long-term-memory-related synaptic plasticity involves bidirectional regulation of gene expression and chromatin structure. Cell 2002; 111: 483-493.

9 Narlikar Geeta J, Sundaramoorthy R, Owen-Hughes T. Mechanisms and functions of ATP-dependent chromatin-remodeling enzymes. Cell 2013; 154: 490-503.

10 Lipsky RH. Epigenetic mechanisms regulating learning and long-term memory. Int J Dev Neurosci 2013; 31: 353-358.

11 Tonegawa S, Pignatelli M, Roy DS, Ryan TJ. Memory engram storage and retrieval. Curr Opin Neurobiol 2015; 35: 101-109.

12 Roberson ED, Sweatt JD. A biochemical blueprint for long-term memory. Learn Mem 1999; 6: 381-388.

13 Day JJ, Sweatt JD. DNA methylation and memory formation. Nat Neurosci 2010; 13: 1319-1323.

14 Volianskis A, France G, Jensen MS, Bortolotto ZA, Jane DE, Collingridge $\mathrm{GL}$. Long-term potentiation and the role of $\mathrm{N}$-methyl-D-aspartate receptors. Brain Res 2015; 1621: 5-16.

15 Bliss TV, Lomo T. Long-lasting potentiation of synaptic transmission in the dentate area of the anaesthetized rabbit following stimulation of the perforant path. J Physiol 1973; 232: 331-356.

16 Lisman J, Yasuda R, Raghavachari S. Mechanisms of CaMKII action in long-term potentiation. Nat Rev Neurosci 2012; 13: 169-182.

17 Grover LM, Kim E, Cooke JD, Holmes WR. LTP in hippocampal area CA1 is induced by burst stimulation over a broad frequency range centered around delta. Learn Mem 2009; 16: 69-81.

18 Peng S, Zhang Y, Zhang J, Wang H, Ren B. ERK in learning and memory: a review of recent research. Int J Mol Sci 2010; 11: 222-232.

19 Vickers CA, Wyllie DJ. Late-phase, protein synthesis-dependent long-term potentiation in hippocampal CA1 pyramidal neurones with destabilized microtubule networks. Br J Pharmacol 2007; 151: 1071-1077.

20 Holtmaat AJ, Trachtenberg JT, Wilbrecht L, Shepherd GM, Zhang X, Knott GW et al. Transient and persistent dendritic spines in the neocortex in vivo. Neuron 2005; 45: 279-291.

21 Dudai Y. The neurobiology of consolidations, or, how stable is the engram? Annu Rev Psychol 2004; 55: 51-86. 
22 Restivo L, Vetere G, Bontempi B, Ammassari-Teule M. The formation of recent and remote memory is associated with time-dependent formation of dendritic spines in the hippocampus and anterior cingulate cortex. J Neurosci 2009; 29: 8206-8214.

23 Miller CA, Sweatt JD. Covalent modification of DNA regulates memory formation. Neuron 2007; 53: 857-869.

24 Miller CA, Gavin CF, White JA, Parrish RR, Honasoge A, Yancey CR et al. Cortical DNA methylation maintains remote memory. Nat Neurosci 2010; 13: 664-666.

25 Yu N-K, Baek SH, Kaang B-K. DNA methylation-mediated control of learning and memory. Mol Brain 2011; 4: 1-9.

26 Levenson JM, Roth TL, Lubin FD, Miller CA, Huang IC, Desai P et al. Evidence that DNA (cytosine-5) methyltransferase regulates synaptic plasticity in the hippocampus. J Biol Chem 2006; 281: 15763-15773.

27 Klose RJ, Bird AP. Genomic DNA methylation: the mark and its mediators. Trends Biochem Sci 2006; 31: 89-97.

28 Guo JU, Su Y, Zhong C, Ming G-I, Song H. Emerging roles of TET proteins and 5-Hydroxymethylcytosines in active DNA demethylation and beyond. Cell Cycle 2011; 10: 2662-2668.

29 Rudenko A, Dawlaty Meelad M, Seo J, Cheng Albert W, Meng J, Le T et al. Tet1 is critical for neuronal activity-regulated gene expression and memory extinction. Neuron 79: 1109-1122.

30 Sarkar S, Abujamra AL, Loew JE, Forman LW, Perrine SP, Faller DV. Histone deacetylase inhibitors reverse $\mathrm{CpG}$ methylation by regulating DNMT1 through ERK signaling. Anticancer Res 2011; 31: 2723-2732.

31 Rountree MR, Bachman KE, Herman JG, Baylin SB. DNA methylation, chromatin inheritance, and cancer. Oncogene 2001; 20: 3156-3165.

32 Kriaucionis S, Bird A. DNA methylation and Rett syndrome. Hum $\mathrm{Mol}$ Genet 2003; 12: R221-R227.

33 Oliveira AMM, Hemstedt TJ, Bading H. Rescue of aging-associated decline in Dnmt3a2 expression restores cognitive abilities. Nat Neurosci 2012; 15: 1111-1113.

34 Monsey MS, Ota KT, Akingbade IF, Hong ES, Schafe GE. Epigenetic alterations are critical for fear memory consolidation and synaptic plasticity in the lateral amygdala. PLOS ONE 2011; 6: e19958.

35 Zovkic IB, Guzman-Karlsson MC, Sweatt JD. Epigenetic regulation of memory formation and maintenance. Learn Mem 2013; 20: 61-74.

36 Zovkic IB, Sweatt JD. Epigenetic mechanisms in learned fear: implications for PTSD. Neuropsychopharmacology 2013; 38: 77-93.

37 Inaba H, Tsukagoshi A, Kida S. PARP-1 activity is required for the reconsolidation and extinction of contextual fear memory. Mol Brain 2015; 8: 1-15.

38 Doerks T, Copley RR, Schultz J, Ponting CP, Bork P. Systematic identification of novel protein domain families associated with nuclear functions. Genome Res 2002; 12: 47-56.

39 Levenson JM, O'Riordan KJ, Brown KD, Trinh MA, Molfese DL, Sweatt JD. Regulation of histone acetylation during memory formation in the hippocampus. J Biol Chem 2004; 279: 40545-40559.

40 Koshibu K, Graff J, Beullens M, Heitz FD, Berchtold D, Russig H et al. Protein phosphatase 1 regulates the histone code for long-term memory. J Neurosci 2009; 29: 13079-13089.

41 Graff J, Woldemichael BT, Berchtold D, Dewarrat G, Mansuy IM. Dynamic histone marks in the hippocampus and cortex facilitate memory consolidation. Nat Commun 2012; 3: 991.

42 Alarcón JM, Malleret G, Touzani K, Vronskaya S, Ishii S, Kandel ER et al. Chromatin acetylation, memory, and LTP are impaired in $\mathrm{CBP}^{+/}-$mice: a model for the cognitive deficit in rubinstein-taybi syndrome and its amelioration. Neuron 2004; 42: 947-959.

43 Hong L, Schroth GP, Matthews HR, Yau P, Bradbury EM. Studies of the DNA binding properties of histone $\mathrm{H} 4$ amino terminus. Thermal denaturation studies reveal that acetylation markedly reduces the binding constant of the H4 'tail' to DNA. J Biol Chem 1993; 268: 305-314.

44 Bottomley MJ. Structures of protein domains that create or recognize histone modifications. EMBO Rep 2004; 5: 464-469.

45 Chen J, Ghazawi FM, Li Q. Interplay of bromodomain and histone acetylation in the regulation of p300-dependent genes. Epigenetics 2010; 5: 509-515.

46 Jarome TJ, Helmstetter FJ. Protein degradation and protein synthesis in long-term memory formation. Front Mol Neurosci 2014; 7: 61

47 Igaz LM, Vianna MRM, Medina JH, Izquierdo I. Two time periods of hippocampal mRNA synthesis are required for memory consolidation of fear-motivated learning. J Neurosci 2002; 22: 6781-6789.

48 Vecsey CG, Hawk JD, Lattal KM, Stein JM, Fabian SA, Attner MA et al. Histone deacetylase inhibitors enhance memory and synaptic plasticity via
CREB: CBP-dependent transcriptional activation. J Neurosci 2007; 27: $6128-6140$

49 Roth SY, Denu JM, Allis CD. Histone acetyltransferases. Annu Rev Biochem 2001; 70: 81-120.

50 Delcuve GP, Khan DH, Davie JR. Roles of histone deacetylases in epigenetic regulation: emerging paradigms from studies with inhibitors. Clin Epigenetics 2012; 4: 1-13.

51 Guan J-S, Haggarty SJ, Giacometti E, Dannenberg J-H, Joseph N, Gao J et al. HDAC2 negatively regulates memory formation and synaptic plasticity. Nature 2009; 459: 55-60.

52 Yin JC, Tully T. CREB and the formation of long-term memory. Curr Opin Neurobiol 1996; 6: 264-268.

53 Hosoda $\mathrm{H}$, kato $\mathrm{K}$, Asano $\mathrm{H}$, Ito $\mathrm{M}$, Kato $\mathrm{H}$, Iwamoto T et al. CBP/p300 is a cell type-specific modulator of CLOCK/BMAL1-mediated transcription. $\mathrm{Mol}$ Brain 2009; 2: 1-18.

54 Roelfsema JH, White SJ, Ariyürek Y, Bartholdi D, Niedrist D, Papadia F et al. Genetic heterogeneity in rubinstein-taybi syndrome: mutations in both the CBP and EP300 genes cause disease. Am J Hum Genet 2005; 76: 572-580.

55 Bredy TW, Wu H, Crego C, Zellhoefer J, Sun YE, Barad M. Histone modifications around individual BDNF gene promoters in prefrontal cortex are associated with extinction of conditioned fear. Learn Mem 2007; 14: 268-276.

56 Korzus E, Rosenfeld MG, Mayford M. CBP histone acetyltransferase activity is a critical component of memory consolidation. Neuron 2004; 42 . 961-972.

57 Vermeulen M, Mulder KW, Denissov S, Pijnappel WW, van Schaik FM, Varier RA et al. Selective anchoring of TFIID to nucleosomes by trimethylation of histone H3 lysine 4. Cell 2007; 131: 58-69.

58 Gupta S, Kim SY, Artis S, Molfese DL, Schumacher A, Sweatt JD et al. Histone methylation regulates memory formation. J Neurosci 2010; 30: 3589-3599.

59 Jarome TJ, Lubin FD. Histone lysine methylation: critical regulator of memory and behavior. Rev Neurosci 2013; 24: 375-387.

60 Kerimoglu C, Agis-Balboa RC, Kranz A, Stilling R, Bahari-Javan S, Benito-Garagorri $\mathrm{E}$ et al. Histone-methyltransferase MLL2 (KMT2B) is required for memory formation in mice. J Neurosci 2013; 33: 3452-3464.

61 Greer EL, Shi Y. Histone methylation: a dynamic mark in health, disease and inheritance. Nat Rev Genet 2012; 13: 343-357.

62 Wagner T, Jung M. New lysine methyltransferase drug targets in cancer. Nat Biotech 2012; 30: 622-623.

63 Peter CJ, Akbarian S. Balancing histone methylation activities in psychiatric disorders. Trends Mol Med 2011; 17: 372-379.

64 Felsenfeld G, Groudine M. Controlling the double helix. Nature 2003; 421 : 448-453.

65 Chwang WB, O'Riordan KJ, Levenson JM, Sweatt JD. ERK/MAPK regulates hippocampal histone phosphorylation following contextual fear conditioning. Learn Mem 2006; 13: 322-328.

66 Kouzarides T. Chromatin Modifications and Their Function. Cell 2007; 128: 693-705.

67 Dias BG, Ressler KJ. Parental olfactory experience influences behavior and neural structure in subsequent generations. Nat Neurosci 2014; 17: 89-96.

68 Gapp K, Jawaid A, Sarkies P, Bohacek J, Pelczar P, Prados J et al. Implication of sperm RNAs in transgenerational inheritance of the effects of early trauma in mice. Nat Neurosci 2014; 17: 667-669.

69 Berger SL. Histone modifications in transcriptional regulation. Curr Opin Genetics Dev 2002; 12: 142-148.

70 Scourzic L, Mouly E, Bernard OA. TET proteins and the control of cytosine demethylation in cancer. Genome Med 2015; 7: 1-16.

71 Ng CW, Yildirim F, Yap YS, Dalin S, Matthews BJ, Velez PJ et al. Extensive changes in DNA methylation are associated with expression of mutant huntingtin. Proc Natl Acad Sci USA 2013; 110: 2354-2359.

72 Jia $\mathrm{H}$, Morris CD, Williams RM, Loring JF, Thomas EA. HDAC inhibition imparts beneficial transgenerational effects in Huntington's disease mice via altered DNA and histone methylation. Proc Natl Acad USA 2015; 112 : E56-E64.

73 Jakovcevski M, Akbarian S. Epigenetic mechanisms in neurological disease. Nat Med 2012; 18: 1194-1204.

74 Alisch RS, Wang T, Chopra P, Visootsak J, Conneely KN, Warren ST. Genome-wide analysis validates aberrant methylation in fragile $X$ syndrome is specific to the FMR1locus. BMC Med Genet 2013; 14: 1-7.

75 Lopez-Atalaya JP, Gervasini C, Mottadelli F, Spena S, Piccione M, Scarano $\mathrm{G}$ et al. Histone acetylation deficits in lymphoblastoid cell lines 
from patients with Rubinstein-Taybi syndrome. J Med Genet 2012; 49: 66-74.

76 De Jager PL, Srivastava G, Lunnon K, Burgess J, Schalkwyk LC, Yu L et al. Alzheimer's disease: early alterations in brain DNA methylation at ANK1, BIN1, RHBDF2 and other loci. Nat Neurosci 2014; 17: $1156-1163$

77 Ladd-Acosta C, Hansen KD, Briem E, Fallin MD, Kaufmann WE, Feinberg AP. Common DNA methylation alterations in multiple brain regions in autism. Mol Psychiatry 2014; 19: 862-871.

78 Park G, Tan J, Garcia G, Kang Y, Salvesen G, Zhang Z. Regulation of histone acetylation by autophagy in parkinson disease. J Biol Chem 2016; 291: 3531-3540. cc)(1)(2) This work is licensed under a Creative Commons Attribution-NonCommercial-ShareAlike

4.0

International License. The images or other third party material in this article are included in the article's Creative Commons license, unless indicated otherwise in the credit line; if the material is not included under the Creative Commons license, users will need to obtain permission from the license holder to reproduce the material. To view a copy of this license, visit http://creativecommons.org/licenses/by-nc-sa/4.0/ 